\title{
Task-specific sensor settings for electro-optical systems in a marine environment
}

\author{
Piet B.W. Schwering, Sebastiaan P. van den Broek, Rob A.W. Kemp, Henk A. Lensen, \\ TNO Defence, Security and Safety, P.O. Box 96864, 2509 JG The Hague, The Netherlands, \\ piet.schwering@tno.nl
}

\begin{abstract}
Present-day naval operations take place in coastal environments as well as narrow straits all over the world. Coastal environments around the world are exhibiting a number of threats to naval forces. In particular, a large number of asymmetric threats can be present in environments with cluttered backgrounds as well as rapidly varying atmospheric conditions. The automatic detection of small targets by electro-optical systems may be hampered by small surface structure variations at the surface and near the horizon.

In current electro-optical sensor systems processing of imagery is seldom task-specific. Using task-specific settings of sensors, processing and fusion, can improve the performance of electro-optical systems dramatically. This paper discusses the effect of dynamic sensor settings as function of specific tasks and environmental parameters and how these can play a role in the management of sensors in a naval application. In addition, a series of experiments with different targets are presented to demonstrate the benefit of sensor management. Some sensor management approaches for application in infrared systems are discussed.
\end{abstract}

Keywords: Adaptive Sensor Management, Sensor Settings, Sensor Tasks, Panoramic Vision, Detection, Image Enhancement, Image Fusion, Image Stitching.

\section{INTRODUCTION}

More and more, coastal environments are the area of operation of naval forces. All over the world, operations in narrow straits and bay environments cause interaction with small unknown surface vessels. While targets in these waters could be smugglers, additionally, large numbers of asymmetric threats, such as pirates, could be present in environments with highly cluttered backgrounds as well as rapidly varying atmospheric conditions. In the case of anti-piracy operations, both mothership and attack crafts show different signature and track. Inbound targets, such as fast incoming jetski, are likely to make sudden maneuvers and originate from high risk areas. Figure 1 shows some small surface targets that may occur in a harbor environment.

Threat contrasts may be low and varying in littoral environments, and the amount of background clutter can be severe. Electro-optical sensors, used for detection and classification, should be optimized in order to increase reaction time. The assessment of the classification task is important for this procedure.

Present-day electro-optical sensor suites are used for diverse tasks as detection, classification and identification ${ }^{1}$. These inherently passive systems, such as infrared and visible light sensors, can definitely improve situation awareness. For application over long range, elevated sensor masts are best suited for the surveillance task and improvement of the operational picture. Effective combination of electro-optical sensor technology and advanced signal processing will lead to new system concepts. Of greatest importance is the analysis of threat information from the high spatial-resolution imagery recorded with different sensors. Since electro-optical sensors do not have all-weather capability the performance degradation in adverse scenarios, such as caused by the varying conditions in coastal environments, can be relatively high. The use of adaptive sensor management techniques can support the effective application of electro-optical sensors in these environments. As part of the littoral scenario, besides the naval scenario the observation of threats in land scenarios is also essential.

Sensors, and Command, Control, Communications, and Intelligence (C3I) Technologies for Homeland Security and Homeland Defense IX, edited by Edward M. Carapezza, Proc. of SPIE Vol. 7666, 76662I · @ 2010 SPIE · CCC code: 0277-786X/10/\$18 · doi: 10.1117/12.851140 
Typically, detection algorithms make use of different kinds of target features, such as hot spots, motion, and structure. Due to the variation in environment, algorithms based on adaptive sensor management are beneficial in the surveillance process. Incorporation of these algorithms into new types of electro-optical (EO) system concepts, based on infrared and visible light systems as well as systems based on sensor fusion, is foreseen.

In current electro-optical sensor systems processing of imagery is seldom task-specific. Using task-specific settings of sensors, processing and fusion can improve the performance of electro-optical sensor systems dramatically. This paper discusses the effect of dynamic sensor settings as function of specific tasks and environmental parameters and how these can play a role in the management of sensors in a naval application. In addition, results from a series of experiments with different targets are presented to demonstrate the benefit of sensor management.

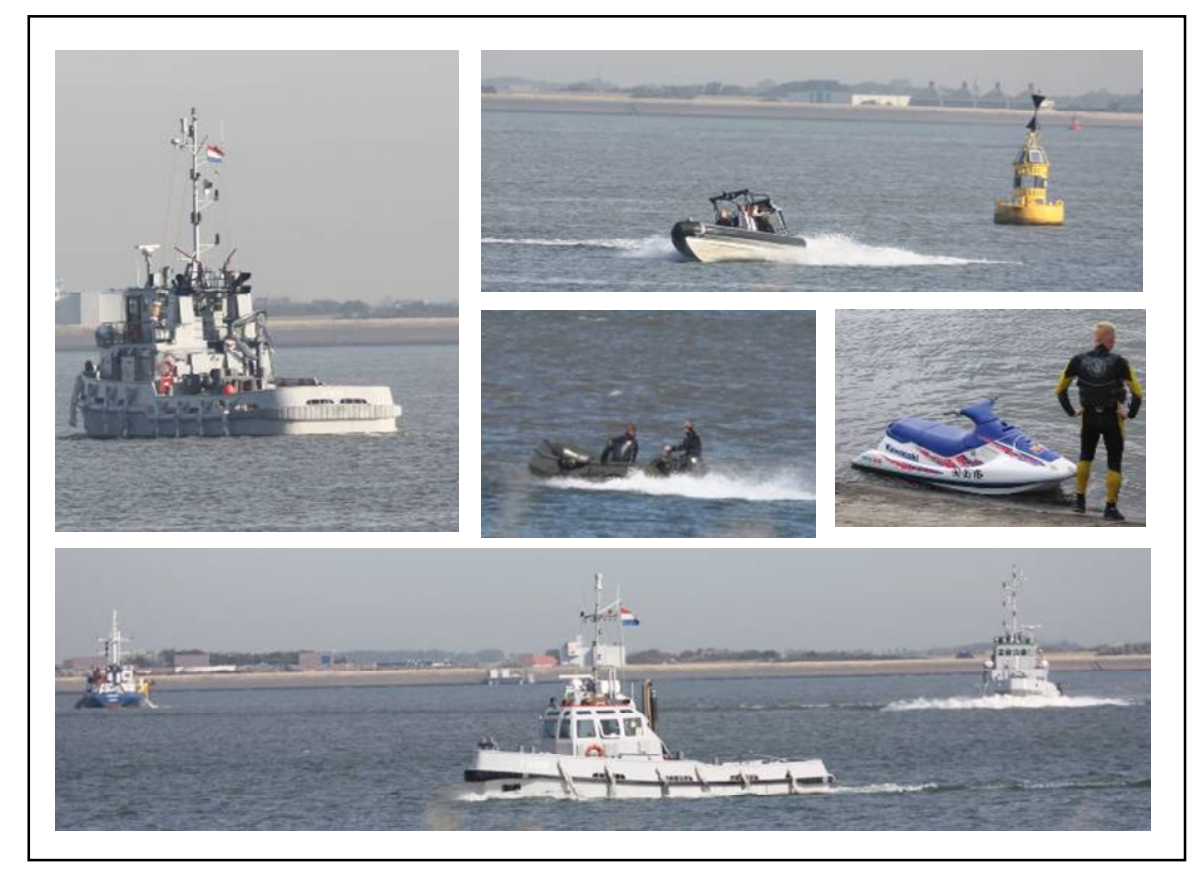

Figure 1 Example of harbor targets during the Den Helder 2009 measurement campaign.

This paper is structured in such a way that it describes the sensor management process starting from a higher operational level, followed by the technical sensors and processing tasks, and ending with detailed operation and algorithms for sensor management. Hence, the next section describes the operational picture compilation for the naval domain, followed in Section 3 by sensor and processing aspects. Section 4 continues with sensor management, the bulk of this paper. Conclusions are gathered in Section 5.

\section{OPERATIONAL PICTURE COMPILATION IN THE NAVAL DOMAIN}

Achieving situation awareness is one of the most important goals in a military mission. Knowing what objects are surrounding you and what the intent of these objects is, is crucial for the decision to go into action, like questioning, boarding or engaging these objects. To achieve situation awareness all available information has to be fused in order to get the right "picture", or Common Operational Picture (COP), of the operations area.

During the operational picture compilation task the area is searched for objects. In case a COP has already been built, a priori information and intelligence of the objects might be available that determine the sensor action. Hence, the task is then to reconfirm the presence of the object and to update the information related to the object. 
Detection and reconfirmation is performed by sensors on board the own ship as well as by sensors of other platforms in the force. Once an object has been detected it is called a contact, which can be automatically tracked. In order to improve the operational picture track information is enriched by additional object properties.

Electro-optical, InfraRed Search and Track systems (IRST), Forward Looking InfraRed cameras (FLIR), radar and Electronic Support Measures (ESM) for example can 'measure' and verify certain object features, and use these to compile and improve the COP. Based on this COP, a priori set identification criteria (idcrits) and rules of engagement (ROE), the user judges the situation and can decide to go into action ${ }^{2}$.

\section{Search and Detect}

During the search and detect task the areas of interest within the operations area are scanned by sensors dedicated to this task. Detection of objects depends on the sensor characteristics, the object features as well as the environment. Sensor settings may be adjusted depending on the expected features of the object and the environment.

$\underline{\text { Track }}$

In a search and track system a track will be initiated automatically after the detection of the object. Information about the object is derived from these tracks. Additional object measurements may be required to get more accurate information on the object's position, movement and intent, which can help to recognize, classify and identify the object.

\section{$\underline{\text { Recognition }}$}

During the recognition and type classification task the type of the object is determined. Recognition can be done at a global level e.g. to determine whether the object is military or civil, or at a more specific level e.g. to determine whether the object is a jetski or a Rigid Hull Inflatable Boat (RHIB).. The object can be recognized based on measurements from various sensors in the force, like radar, IRST (Infrared Search and Track), IFF (Identification Friend or Foe) or AIS (Automatic Identification System).

\section{$\underline{\text { Identification (Friend or Foe) }}$}

During the identification task the object is categorized in groups, comparing information to the identification criteria. Identity categories are friendly, neutral, unknown, suspect, and hostile. Identification of objects may be derived from the object's radar or IRST tracks and/or determined by different sensors like FLIR, IFF, AIS, ESM or visual identification by an operator.

\section{SENSORS AND PROCESSING}

Operational tasks in the naval domain as described in the previous section start with sensor data and other information (for example from intelligence) and end with identifying threats, leading to a better situational awareness, and yielding information on which it is decided whether or how to act against it. This whole process can be done by an operator, viewing the images provided by electro-optical and other sensors, and controlling their parameters. But parts of the process can be taken over by algorithms, as shown schematically in figure 2. An operator can do recognition visually, or use the information extraction results of autonomous detection and tracking processes. For identification, all information obtained in different ways needs to be checked and compared to the identification criteria, to assess whether the object is really a threat or a false alarm. The different processing steps are listed below.

\section{Image improvement}

Techniques as image stabilization, noise reduction, resolution enhancement, and contrast enhancement can make it easier for an operator or process to observe an object of interest.

\section{Detection and clutter reduction}

Automatic detection of objects can result in earlier detection of objects that are difficult to see, or make it possible to cover a larger search area. Clutter reduction makes sure no objects have to be checked that are due to false alarms. 


\section{Tracking}

Tracking provides kinematic information about an object, and more importantly links measurements in time, so information determined at one time can be used at a later time. This also allows temporal clutter reduction, evaluation of behavior, such as sudden course changes, maneuverability or whether an object is incoming at high speed.

\section{Feature extraction}

Providing automatically determined features linked to a track may help prioritizing tracks as possible threats (by eliminating types), or help an operator decide what an object is.

\section{Type classification}

Automatic indication of what type of object a detected target could be, helps prioritizing attention to different tracks, or automatically rules out objects as being a threat. Even less specific type categorization, such as for example 'fishing trailer', can help an operator make a classification needed for identification, in a process called aided target recognition.

\section{Identification}

All information known about an object is checked with identification criteria, which can come from automatic processing in earlier steps, from intelligence, or from the operator. A semi-automatic process allows the combination of information of many sources, while still giving an operator the final decision.

A multi-sensor concept could use a sensor with large field-of-regard, such as a 360 degree staring IR system or radar, for automatic detection ${ }^{3}$. After detection, clutter reduction and tracking, a higher resolution camera system could be queued to tracks and allow determination of features for type classification and finally identification. Besides input from an operator at different levels (i.e., putting priority on a track to view, or adding a classification label), there are different points at which sensor settings are critical to the process. Sensor management is the automatic adjustment of settings to best fit the changing tasks in a changing environment. To support this process, known information about objects can be compared to the required information, and sensors are adjusted to best fit the uncertainty in information that is required for identification. An example of such a process is given in paper ${ }^{2}$. Besides selection of sensors, dynamically adapting sensor settings is important in varying circumstances. These occur in coastal and harbor environments, where atmospheric and background conditions have a direct influence on target contrast in EO sensors. This means that in such an environment the sensor management should adapt to specific conditions in order to optimize overall system performance.

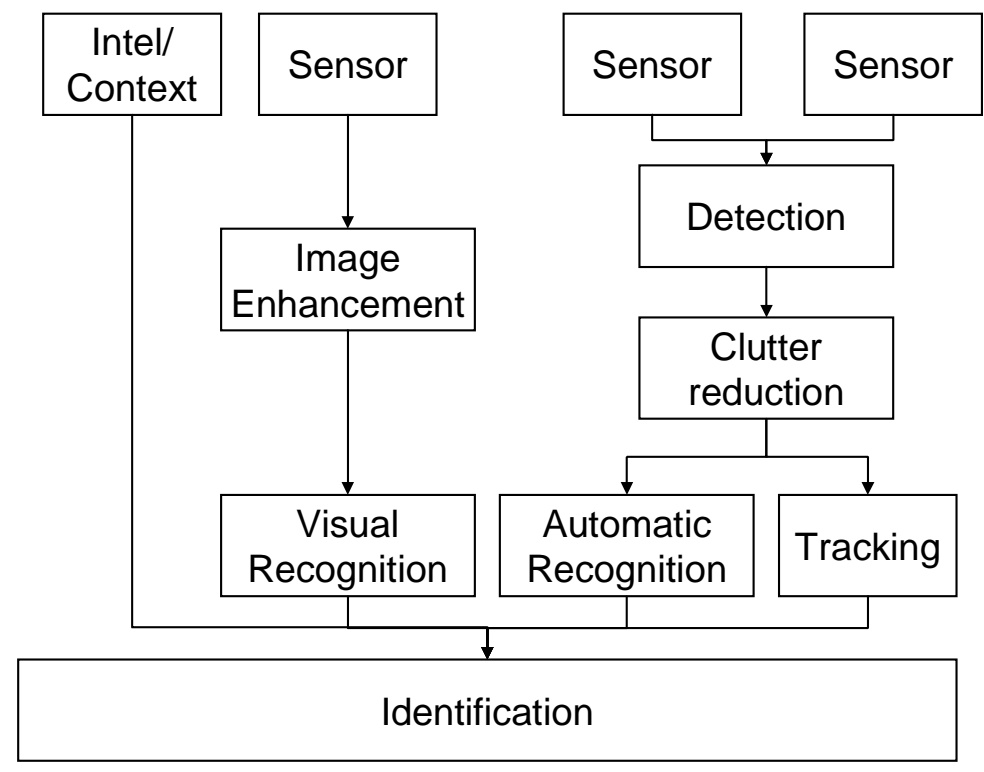

Figure 2 Schematic example of a threat assessment process with automatic processing and visual classification by an operator 


\section{SENSOR MANAGEMENT}

The main task of an image formation sensor (i.e., cameras) is to generate images for (human) surveillance purposes, whereas the task of a sensor for autonomous target detection is to detect (air or surface) targets without the need for a human observer. In order to obtain optimum task performance the sensors and their processing algorithms (type, parameters) should be tuned to the application, threat type, environmental conditions and required performance. For the surveillance task of the image formation sensor this can be obtained e.g. by generating a high quality image with a large field of view (FOV), high contrast, high resolution and low noise. For the autonomous target detection task this can be obtained e.g. by optimizing the detection probability and tolerable false alarm rate.

A prerequisite to sensor management is knowledge about the sensors and processes that are part of the observation or target detection chain. Knowledge about the sensors can help to select the best sensor to carry out the task. Targets, backgrounds and atmosphere can produce different radiation contrasts in different wavelength bands and as function of e.g. the operational condition of the target, weather or area of operation. Therefore, the perceived target-background contrast can differ for each (complementary) sensor. Moreover, specific sensor characteristics as Noise Equivalent Irradiance (NEI), Instantaneous Field-of-View (IFOV), FOV and minimum resolvable temperature difference (MRTD) determine if, when and how reliable a target will be detected or classified. Knowledge about the (sub-) processes in the EO-data processing scheme can help to optimize the processing results for each sub-process

A way in which to improve both capabilities is to add extra sensors that operate in complementary wavelength bands and to adaptively manage the processing algorithms in response to changes in image condition, environment, threat, task and performance requirement. This is of particular importance to multi-sensor target detection or observation systems when one of the sensors performances degrades or counter-measures are deployed.

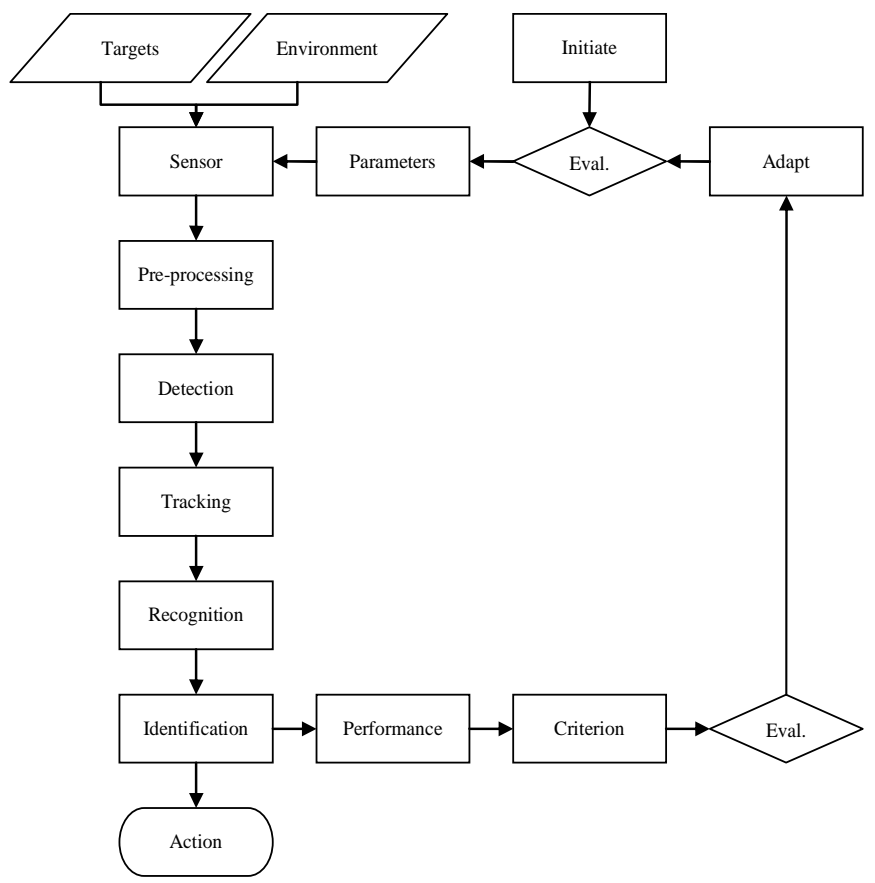

Figure 3 Example of an adaptive sensor management system for a generic processing scheme with a feedback loop. In effect each of the processing blocks, from pre-processing to recognition, require a similar loop as specified for the identification block.

The idea behind Adaptive Sensor Management (ASM) is that electro-optical systems perform better when feedback or information is used from other sensors or from other parts of the processing scheme. In case of sensors for image formation this feedback or information allows for optimization of a single image for observation. In case of a multisensor observation system, feedback or information also enables the observer (or the system) to select the most 
optimized sensor, or a combination of sensors (fusion), to carry out the observation task. Sensors for autonomous target detection like IRST can, based on feedback or information, select the best algorithms with optimized parameters to manage detection performance and false alarm rates. Figure 3 shows a generic approach to a closed loop adaptive sensor management system.

One can think of a Threat Detection Request (TDR) to a sensor management system. Based on target properties and environment conditions, the TDR induces sensor settings adjustments in order to meet the detection criteria for the specific target or range of targets. Since it is likely that a single target is more confidently detected by a system of sensors than by a single sensor, multiple sensors have to be adjusted at the same time.

The following list specifies what sensor settings and algorithm parameters can be adjusted at different levels to optimize the process:

- Choice of sensor: some sensors may provide missing information for a tracked object, where others do not. This also depends on environmental conditions. For example, an infrared camera may provide features about hot spots that a visual light camera can not, but in high humidity conditions and required longer operating ranges, a midwave infrared system is performing better than a long wave infrared camera.

- Automatic sensor setting adjustment: sensor settings can be automatically adjusted to improve the image under given environmental conditions, in order to provide better results in later stages, be it showing a clearer picture to an operator (in combination with image enhancement) or improve detection or feature extraction.

- Detection: based on where threats are expected (i.e., where cost of missing objects is high), sensors could be directed to specifically detect with higher resolution in a given area.

- Queuing: prioritizing tracked objects by whether they may be a threat, taking into account what information (features, type classification, identification) ${ }^{4}$ is inaccurate that might confirm this to be a threat or not. This accuracy also depends on how old information is or whether an operator has confirmed information (such as a type). Sensors may be queued to tracks based on this prioritization to fill in gaps. With automatic queuing also recordings of all tracks could be saved and made available to an operator for viewing.

In order to demonstrate the concept of ASM we were tasked to identify a vessel at a range of approximately $12 \mathrm{~km}$. In this case identification was done by reading the name on the hull of a ship.using a long range camera. The range prediction model EOSTAR ${ }^{5}$ pointed out that (based on the meteorological conditions) severe turbulence could occur and disturb the imagery, hence dominating the image resolution. When target's distance was assessed, with the EOSTAR information the turbulence correction was automatically switched on and parameterized by ASM. Since the ship's name was white-on-grey, local adaptive contrast enhancement was also applied automatically for the ease of reading. Figure 4 shows the actual application of ASM with turbulence correction and contrast enhancement in the above mentioned case.

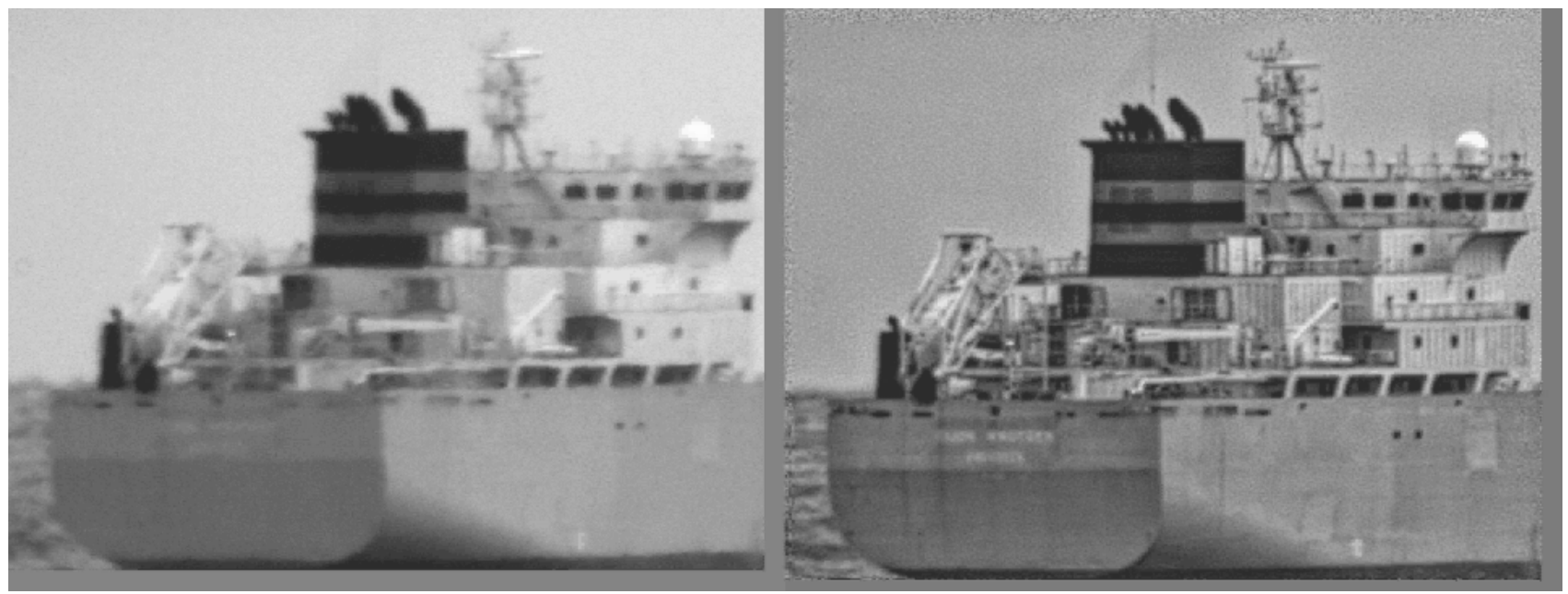

Figure 4 Observing a long distance target (left hand picture is original TV image): turbulence correction switched on (right hand picture) 
In ASM parameters can be set at various stages in the processing chain. Sensor adjustments are limited directly to sensor type selection, sensor settings, such as gain, dynamic range, wavelength filters, tables for non-uniformity correction, etc. Besides availability of a sensor and the ROE, sensor type selection is primarily based on operational task and expected target contrast. In this process an accurate sensor performance model such as EOSTAR is essential. In several cases multiple sensor bands are selected. Image data, such as measured noise and measured dynamic range, can then be used to select or combine image data. Output data amount can also be the limiting factor in a specific task. The operator (or algorithm) can not process more than a certain amount of information. Additionally pre-processing ASM is based on optimizing the images in the first processing stage. The above mentioned turbulence is a typical example of this, but it can also imply for instance the automatic enabling of Dynamic Super Resolution in order to generate more resolution in the images for more distant targets. A full description of potential algorithms of use in the pre-processing stage is given in other papers ${ }^{6,7}$.

In the detection process typically detection thresholds are set based on constant false alarm rate algorithms that adapt quickly to environmental circumstances ${ }^{8}$. In a strongly varying environment care should be taken to limit strong oscillations of the sensor and processing parameters in order to enhance algorithm stability. Furthermore the use of real performance information on the algorithms ROC (Receiver Operator Characteristics), that varies in specific conditions) can be applied to optimize sensor performance in varying circumstances, by adapting detection windows and detection thresholds. For tracking processes similar parameters can be adapted, such as track length for track promotion, track loss, alarm declaration. The ASM process allows for improved performance in more complex scenarios.

If an observer is to look at the imagery, comfortable presentation methods can emerge from the combination of sensor imagery and image improvement. In such cases Sensor Management will combine the best images, apply contrast optimization to the fused result and present them to the observer. In conclusion Adaptive Sensor Management can be fed with a specific task (TDR) in order to obtain target / object features in the observation space of the platform.

The weighed use of images in multiple bands, selecting images that contain relevant information and make that information visible in a better way by fusion, helping an operator. Currently, multi-band and hyperspectral imaging sensors in the thermal infrared are under development. These systems promise significant improvements in military task performance. With these new systems, targets may be distinguished not only on the basis of differences in radiation magnitude, but also on differences in spectral properties. A problem with presenting multi- or hyperspectral imagery to a human observer is the (huge) amount of information. The question is how the data should be made available to the human visual system, i.e. which presentation offers the best information transfer ${ }^{9}$. This also depends on the task at hand (e.g. detection, situational awareness, identification) and the prior information available.

In a previous paper ${ }^{3}$ we used two color representations to visualize a multi-band image of a scene observed during the harbor trial. The multi-band image consisted of four images of a frigate docked in the harbor with a group of people standing on the bow (figure 5).

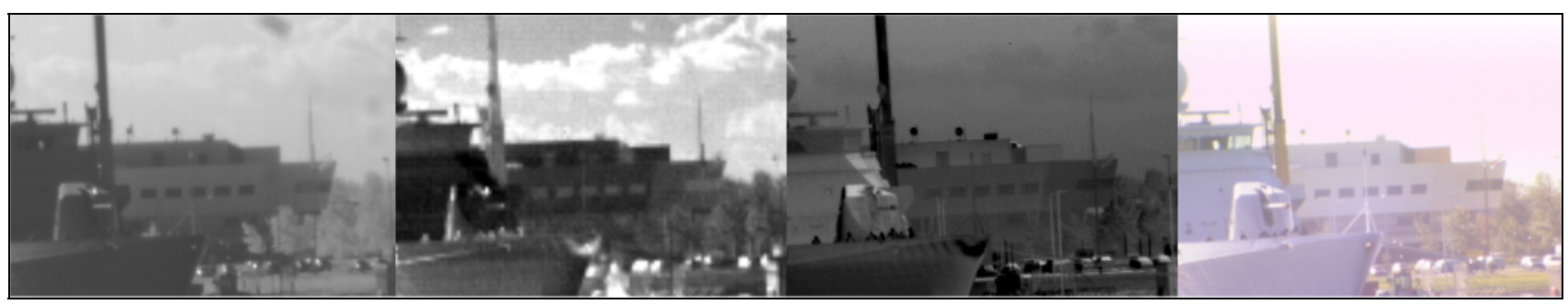

Figure 5 Images of a frigate docked in the harbor with a group of people standing on the bow. The images are acquired by a near infrared camera (first image left), mid-wave infrared camera (second image), long-wave infrared camera (third image) and a color camera (image right).

The four images were acquired by a near infrared camera (image left), a mid-wave infrared camera (second image left), a long-wave infrared camera (third image left) and a color camera (image right). In order to get the color representations first a principal component transformation was carried out. Next, the first three principal components were assigned with hue, saturation and value signals. The result image is depicted in figure 6 (left image). 
In this image there is a clear distinction between the people on the bow (dark dots on the left), the frigate, building, parking lot and the sky. Also sun reflections such as arriving from the parked cars are clearly visible (dark blobs on the lower right). In the right image of figure 6 the hue and saturation were taken from the color image. This color representation appears more natural. Now, the trees and grass are better visible. Both representations can enhance the situation awareness. This shows that selection of the type of fusion process can be very dependent on scenario and environment.

At night, when no daytime image is available, a recently developed technique for adding natural daytime colors to nighttime imagery can be used to enhance situation awareness and create a more intuitive image ${ }^{10}$.

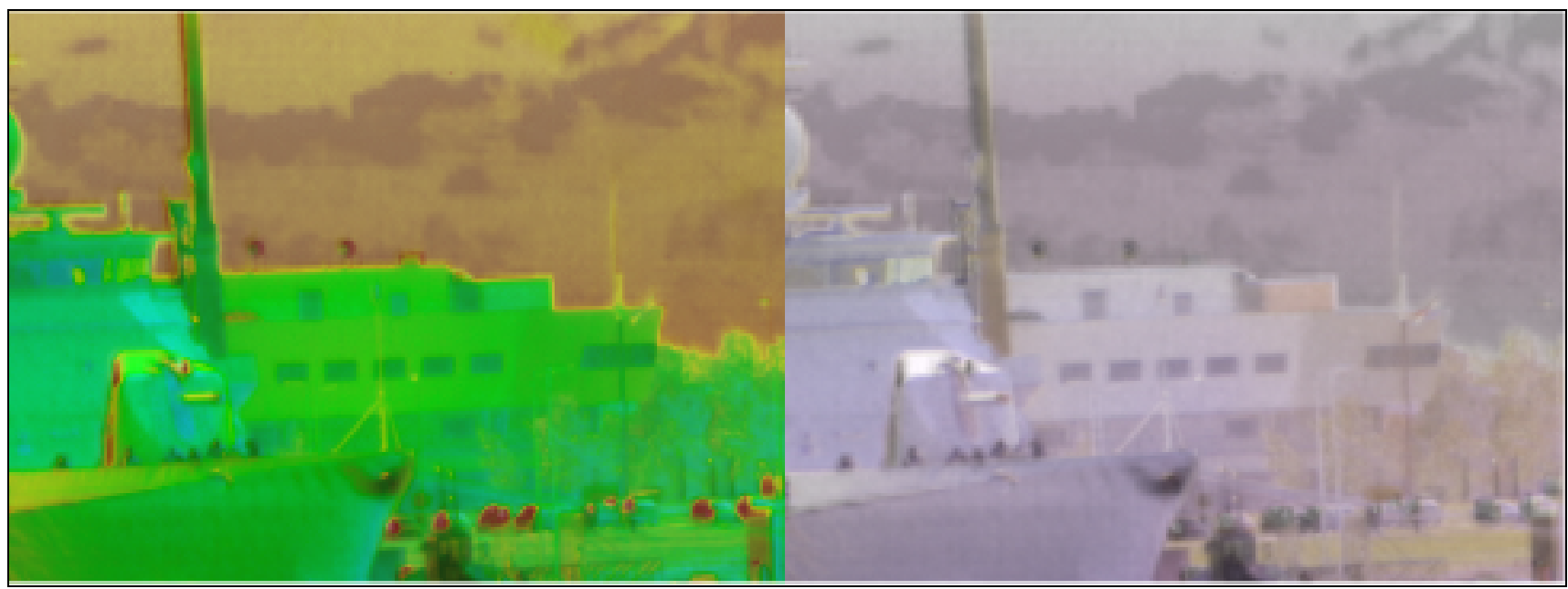

Figure 6 Color representation of a multi-band image using the first three principal components as hue, saturation and value signals (left). The multi-band image combines a near infrared, mid-wave infrared, long-wave infrared and color image. In the image right the hue and saturation is obtained from the color image.

Managing algorithm settings should be done tuning on the required end result, keeping information available as long as possible. Figure 7 shows an example of the combined detection, clutter reduction and tracking. By correctly applying filtering on features to reduce clutter, and using track filtering to remove short-lived tracks, it is possible to use a very sensitive detection, that is able to detect small buoys and a RHIB. The detection techniques applied here are described in the paper by Bouma et $\mathrm{al}^{11}$.

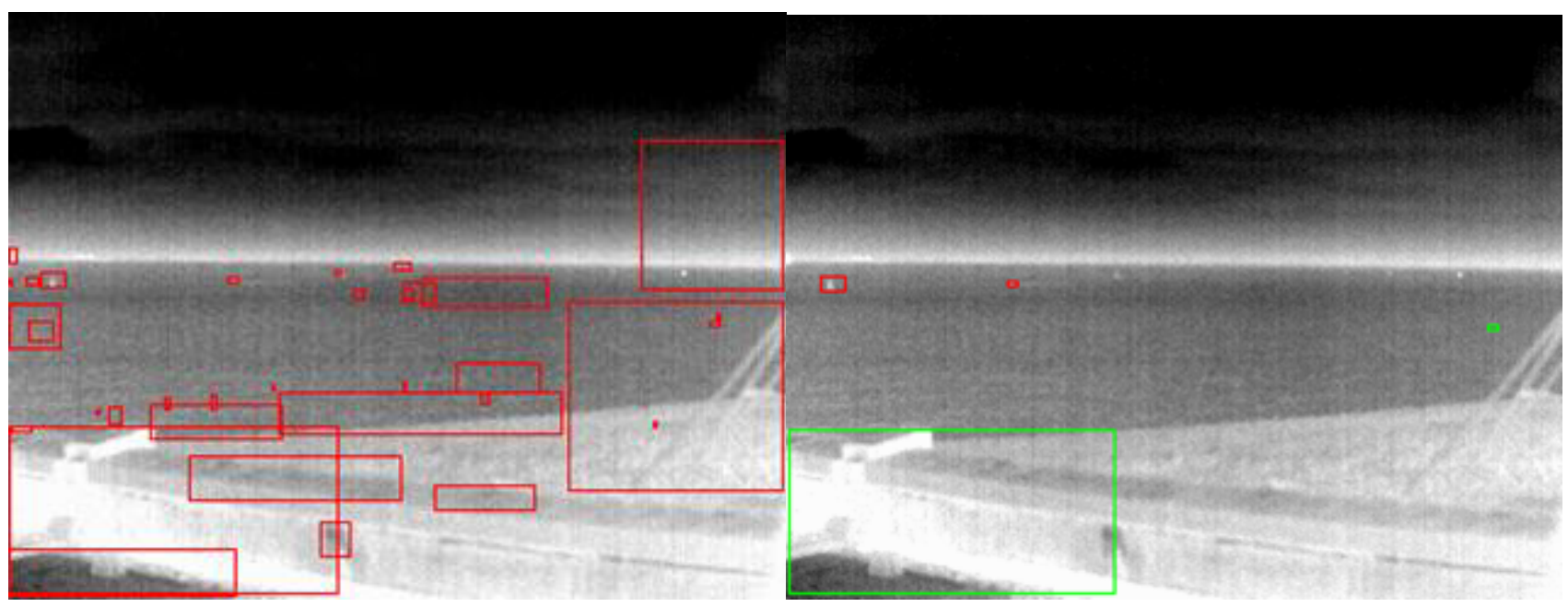

Figure 7 Example of detection with low threshold (left) and the result after clutter reduction and tracking. The remaining red tracks are a buoy and a RHIB. 
Another effective example of helping an operator in his task of situation awareness is by controlling sensors automatically and displaying information in a relevant way, as shown in figure 8. Here a high resolution camera is queued to the detections of a small target (RHIB) in a low-resolution staring camera system. The resulting highresolution image is aligned with the low resolution image and positioned to provide both a good overview of the surroundings, while making the small target visible, and provide more detail.

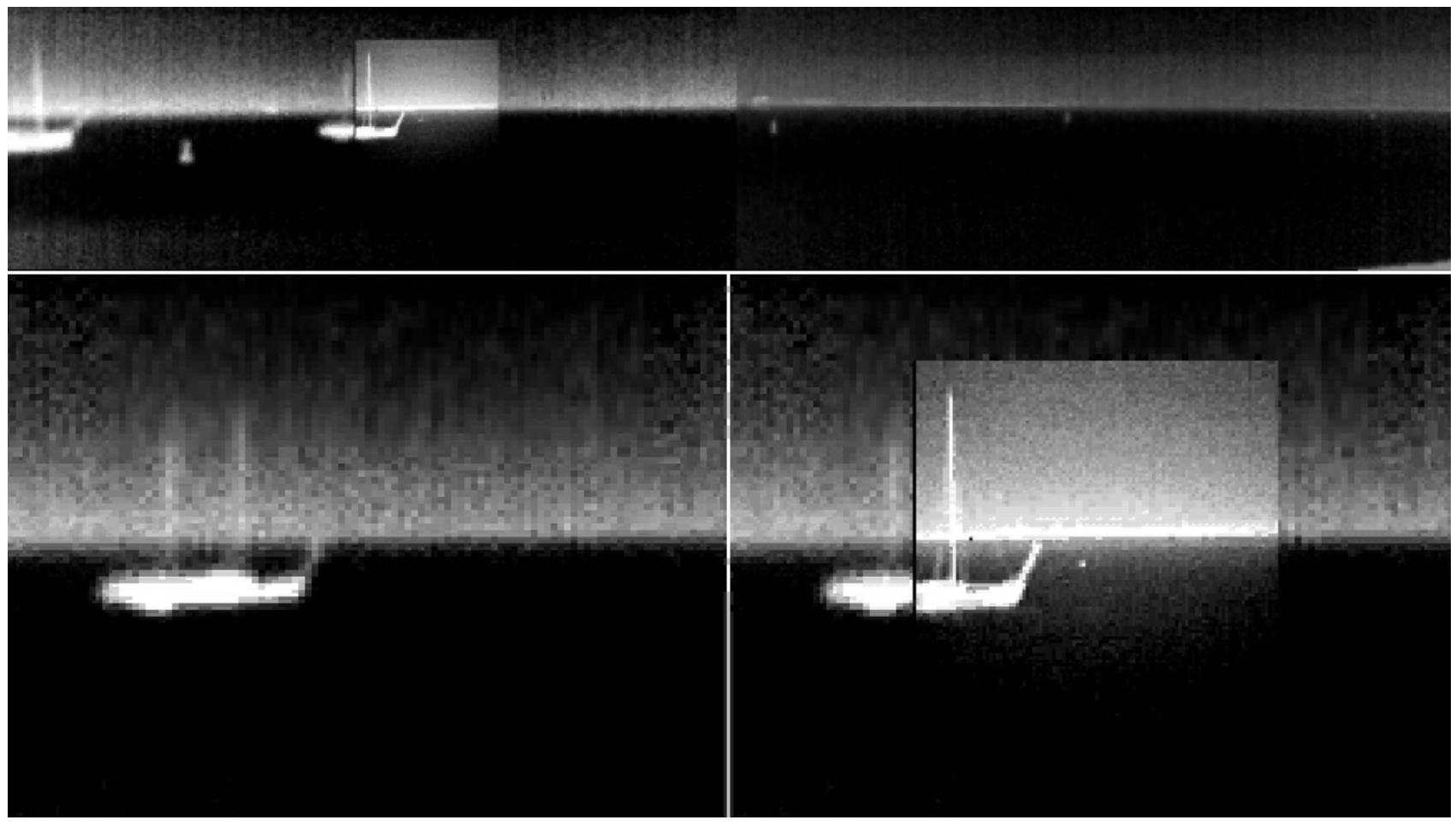

Figure 8 Stitching of a high resolution image in a low resolution overview. Top: two low resolution camera images stitched together, with a high resolution image positioned in the correct location. Bottom: close up of the display around the high resolution camera image, left without and right with the high resolution image. More detail is visible, and the RHIB in the centre is clearly visible in the high resolution insert.

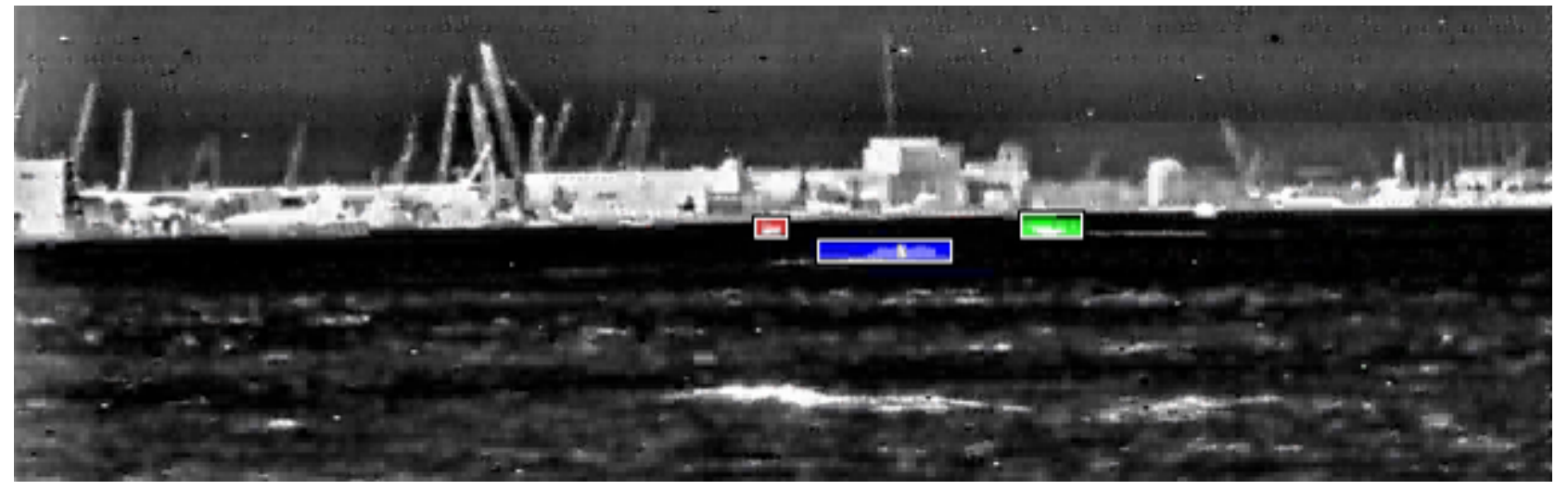

Figure 9 Frame from a sequence processed by the processing chain described in section 3, including type classification based on global descriptors. Color labels indicate classification type. Water taxi (red, left), jet-ski (blue, middle) and RHIB (green, right) are all classified correctly. 
Automatic type classification ${ }^{4,12}$ can be used to discriminate amongst different types of vessels. An example is shown in figure 9, where automatically detected and tracked ships are classified by type and given different color labels. The color labels suite the operator's interpretation of the imagery. Other approaches for type classification, such as silhouette analysis $^{13}$, as well as combination of different approaches, can also be used here.

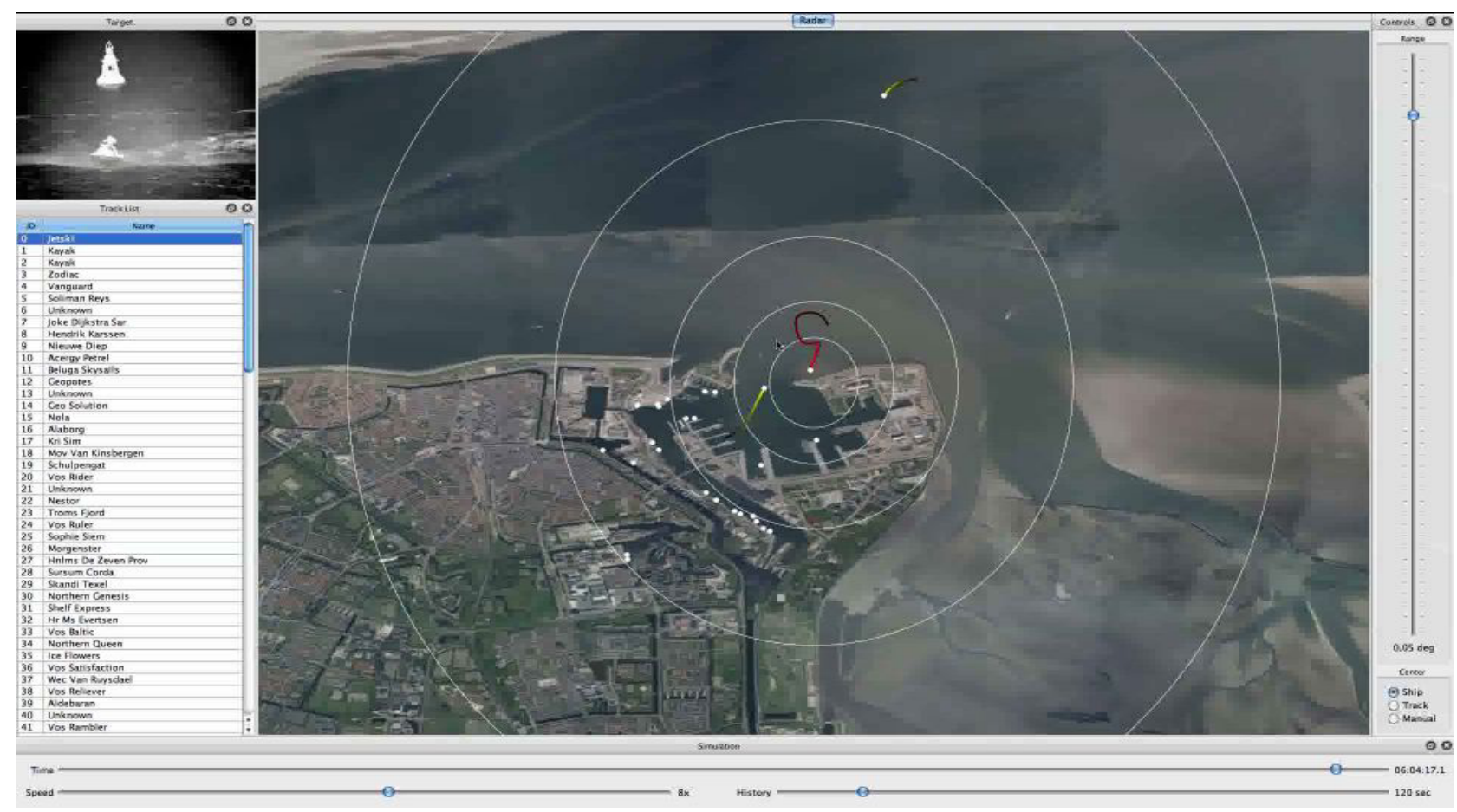

Figure 10 Example of situational awareness software showing identified tracks on a local image map. The operator can select tracks to display recordings that were made of this object at an earlier time (upper left image).

Automatic queuing of a high resolution camera can also be used to keep a visual record of all tracked objects. An operator can review the recordings of tracks of interest to add visual recognition information. Figure 10 shows an example of such an operator view, where a recording of a jet ski is replayed when selecting its track.

In such an approach additional information from a large number of sensors can be used. In an earlier paper ${ }^{14}$ the use of combining different types of sensors is described. In there, radar, infrared and laser imagery from small vessels is combined to obtain improved situational awareness from a scene with small vessels.

With the aim of understanding and optimizing adaptive sensor management (ASM), TNO has executed a number of experiments making use of different electro-optical sensor systems in various wavebands ranging from 0.4 to $14 \mu \mathrm{m}$ on a wide range of potential targets.

Some examples of sensors used during these experiments are given in figure 11. 


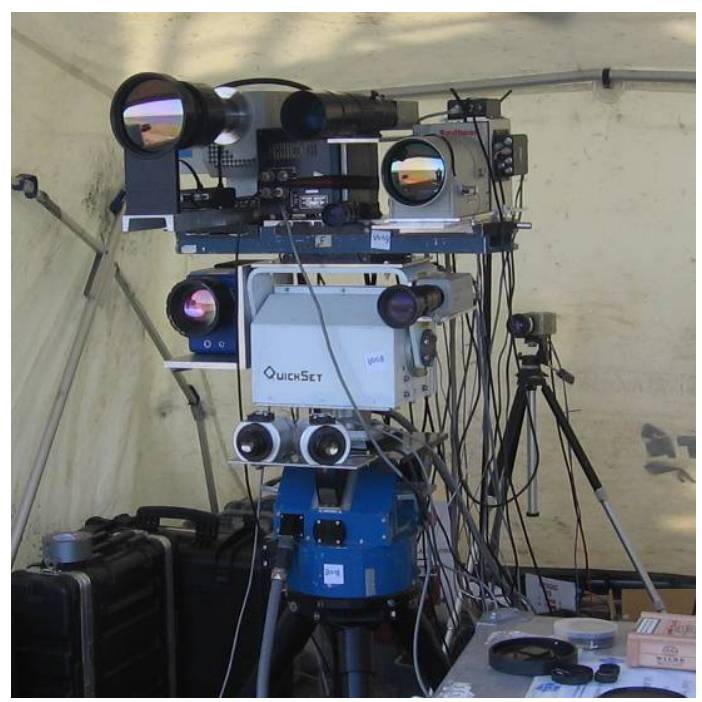

Figure 11 Examples of harbor targets and EO sensor systems during the Den Helder 2009 measurement campaign.

\section{CONCLUSIONS}

In this paper, an overview of the process of sensor management was given, with discussion of several aspects of sensor selection and managing settings of sensors and processing algorithms. It can be concluded that adaptive sensor management is an effective means to optimize sensor performance for a specific operational task in a local environment. It allows for sensor selection as well as for sensor and processing optimization in a (strongly) varying environment. Results from several experiments show that type classification, sensor fusion and the combination with sensor management are essential tools for enhancing situation awareness. The benefit of sensor fusion can be improved by allowing for fusion processes that adapt to the local environment and operational tasks as well. Sensor management takes place at different operational levels, and therefore requires an extensive algorithm that weighs the effect of system settings at all levels on the resulting object identification. To be able to tune such a multi-level sensor management system, extensive training on operationally relevant data is essential.

\section{ACKNOWLEDGEMENTS}

The work for this paper was financially supported in the period 2006-2009 by the Netherlands MoD under the program V602 "Electro-Optical sensor systems". Specific results used in this paper were obtained in the National Technology Project Mafuse (Management and Fusion of Sensors) executed for the Netherlands MoD. 


\section{REFERENCES}

1. Schwering, P.B.W, Broek, S.P. van den, and Iersel, M. van, "EO System Concepts in the Littoral," Proc. SPIE $6542,654230(2007)$

2. van Norden, W., Jong, J. de, Bolderheij, F., and Rothkrantz, L., "Intelligent Task Scheduling in Sensor Networks", Proc. $8^{\text {th }}$ Int. Conf. On Information Fusion, 0-7803-9286-8/05, Philadelphia, USA (2005)

3. Lensen, H.A., Schwering, P.B.W., Broek, S.P. van den, Hollander, R.J.M. den, Mark, W. van der, and Kemp, R.A.W., "' in 'Sensors, and Command, Control, Communications, and Intelligence (C3I) Technologies for Homeland Security and Homeland Defense VIII', ed Carapezza, Edward M., SPIE Vol. 7305 pp. 73050B73050B-16 (2009)

4. Broek, S.P. van den, Bouma, H., Degache, M.A.C., and Burghouts, G., "Discrimination of classes of ships for aided recognition in a coastal environment," Proc. of SPIE Vol. 7335, 73350W (2009)

5. Kunz, G.J., Degache, M.A.C., Moerman, M.M., Eijk, A.M.J. van, Neele, F.P., Doss-Hammel S.M., and Tsintikidis, D., "Status and Developments in EOSTAR, a model to predict IR sensor performance in the marine environment," Proc. SPIE 5572, 101-111 (2004)

6. Schutte, K., Lange, D.J.J. de, and Broek, S.P. van den, "Signal conditioning algorithms for enhanced tactical sensor imagery", Proc. SPIE 5076, 92 (2003)

7. Kemp, R.A.W, de Groot, J.F., Broek, S.P. van den, Lange, D.J.J. de, Dijk, J., and Schwering, P.B.W., "Results of Optical Detection Trials in Harbour Environment," SPIE Proc. 6943, 69430Y (2008)

8. Haddon, J.F., and Boyce, J.F. "Adaptive dynamic scene analysis", in 'Imaging and vision systems: theory, assessment and applications' ISBN 1-59033-033-1, pp 207-234, Nova Science Publishers, Inc., Commack, NY, USA (2001)

9. Schwering, P.B.W., Bekman, H.H.P.Th., and Seijen, H.H. van, "HYBASE: Hyperspectral band selection", Proc SPIE 7334-73 (2009)

10. Toet, A. "Natural Colour Mapping for Mulitband Nightvision Imagery", Information Fusion 4, pp 155-166 (2003)

11. Bouma, H., Lange, D.J.J. de, Broek, S.P. van den, Kemp, R.A.W. and Schwering, P.B.W., "Automatic detection of small surface targets with electro-optical sensors in a harbor environment," Proc. SPIE 7114, 711402 (2008)

12. Broek, S.P. van den, Bouma, H., and Degache, M.A.C., "Discriminating small extended targets at sea from clutter and other classes of boats in infrared and visual light imagery," Proc. SPIE 6969, 69690B (2008)

13. Withagen, P., Schutte, K., Vossepoel, A.M., and Breuers, M., "Automatic classification of ships from infrared (FLIR) images," Proc. SPIE 3720, 180-187 (1999)

14. Broek, A.C. van den, Broek, S.P. van den, Heuvel, J.C. van den, Schwering, P.B.W., and Heijningen, A.W.P. van, "A Multi-sensor scenario for coastal surveillance," Proc. SPIE 6736, 67360P (2007) 